\title{
Evolution of The Angiopoietin-Like Gene Family and Their Role in Lipid Metabolism in Pigs
}

\section{Zibin Zheng}

Wuhan Polytechnic University School of Animal Science and Nutritional Engineering

Wentao Lyu

Zhejiang Academy of Agricultural Sciences

\section{Qihua Hong}

Zhejiang University

\section{Hua Yang}

Zhejiang Academy of Agricultural Sciences

\section{Ying Li \\ Foshan University School of Life Science}

\section{Shengjun Zhao}

Wuhan Polytechnic University School of Animal Science and Nutritional Engineering

\section{Ying Ren}

Wuhan Polytechnic University School of Animal Science and Nutritional Engineering

Yingping Xiao ( $\nabla$ ypxiaozj@hotmail.com )

Zhejiang Academy of Agricultural Sciences https://orcid.org/0000-0002-7949-1126

\section{Research article}

Keywords: Angiopoietin-like protein, Evolution, Expression, Fat metabolize, Jinhua pig

Posted Date: July 19th, 2021

DOl: https://doi.org/10.21203/rs.3.rs-538958/v2

License: (c) (i) This work is licensed under a Creative Commons Attribution 4.0 International License. Read Full License 


\section{Abstract \\ Background}

Lipid metabolism is closely associated with various metabolic diseases, such as obesity, cardiovascular disease, diabetes, and cancer. More importantly, it also affects the carcass quality of animals. Angiopoietin-like protein (Angiopoietin-like, ANGPTL) family members are encoded by 8 genes and play an important role in lipid metabolism and angiogenesis. In this study, we first systematically described the phylogenetic characteristics of pig ANGPTL family genes and identified the critical roles of ANGPTL3, ANGPTL4 and ANGPTL8 in the lipid metabolism of pigs.

\section{Methods}

The amino acid sequence analysis, phylogenetic analysis, and chromosome adjacent gene analysis were performed to identify the ANGPTL gene family in pigs. Furthermore, according to the body weight data from 60 Jinhua pigs, different tissues of 6 pigs with average body weight were used to determine the expression profile of ANGPTL1-8. The ileum, subcutaneous fat, and liver of 8 pigs with distinct fatness were selected to analyze the gene expression of ANGPTL3, ANGPTL4, and ANGPTL8.

\section{Results}

Results showed the sequence length of ANGPTLs in pigs was between 1186 and $1991 \mathrm{bp}$, and the pig ANGPTL family members shared common features with human homologous genes, including the high similarity of the amino acid sequence and chromosome flanking genes. Amino acid sequence analysis showed that ANGPTL1-7 had a highly conserved domain except for ANGPTL8. Phylogenetic analysis showed that each ANGPTL homologous gene was clustered with other mammalian sequences, away from other vertebrates. RT-qPCR analysis showed that ANGPTL family members had different expression patterns in different tissues. ANGPTL3 and ANGPTL8 were mainly expressed in the liver, while ANGPTL4 was expressed in many other tissues, such as the intestine and subcutaneous fat. The expression levels of ANGPTL3 and ANGPTL4 in Jinhua pigs with low propensity for adipogenesis were significantly higher than those of high propensity for adipogenesis.

\section{Conclusions}

These results increase our knowledge about the biological role of the ANGPTL family in this important economic species. In addition, it will also help to better understand the role of ANGPTL3, ANGPTL4 and ANGPTL8 in lipid metabolism of pigs, and provide innovative ideas for develop strategies to improve pig carcass quality.

\section{Introduction}

The Angiopoietin-like protein (ANGPTL) family is a type of secretory protein that plays a variety of roles in lipid metabolism, glucose metabolism, energy metabolism, angiogenesis, and stem cell biology [1]. Excluding ANGPTL8, ANGPTL families are similar to Angiopoietins (ANG) and share common characteristics, including the $\mathrm{N}$-terminal signal sequence (SS) and coiled-coil domain (CCD), that mediates the formation of homo-oligomers, and the C-terminal fibrinogen domain (FReD) that regulates ligand activity [2, 3]. ANGPTL8, an atypical member of the ANGPTL family, consists of 198 amino acids and lacks FReD in humans [4,5]. Disordered lipid metabolism will lead to cardiovascular disease, diabetes, and obesity, and it also affects the meat quality of animals $[6,7]$. 
The investigation on improving meat production as well as meat quality has been pursued by many animal husbandry researchers. Extensive studies confirm that ANGPTL3, ANGPTL4, and ANGPTL8 play an important role in lipid metabolism and are all lipoprotein lipase (LPL) inhibitors $[8,9]$. LPL was originally identified as a triglyceride (TG) scavenging factor lipase [10]. It is produced by cardiomyocytes and adipocytes and is transported by GPIHBP1 protein to the lumen side of capillary endothelial cells [11], where it hydrolyzes TG into fatty acids for being absorbed into tissues $[12,13]$.

ANGPTL3 is a liver-specific secretion factor and is highly expressed in the liver [14]. Its expression is regulated by the liver $X$ receptor (LXR) [15]. The main effect of ANGPTL3 is to inhibit the activity of LPL in the capillaries of adipose tissue and muscle $[8,16]$. Intriguingly, ANGPTL3 plays a major role in promoting the uptake of very low density lipoprotein (VLDL)derived TG into white adipose tissue (WAT) rather than oxidative tissues, such as skeletal muscle, heart brown adipose tissue in the feeding state [17].

ANGPTL4 is also known as fasting-induced adipokine (FIAF) or peroxisome proliferator-activated receptor gamma angiopoietin-related protein (PGAR) $[18,19]$. ANGPTL4 regulates lipid homeostasis and participates in regulating the intestinal microbiota in fat deposition [20,21]. Unlike ANGPTL3, ANGPTL4 can be secreted by adipose tissue, intestinal tissues, liver, skeletal muscle, heart, and other tissues [22]. ANGPTL4 is released as a $50 \mathrm{kD}$ hormone precursor, which is then cut into N-terminal and C-terminal fragments $[13,23]$. The N-terminus of ANGPTL4 acts as a lipoprotein lipase (LPL) inhibitor [22]. Research shows that ANGPTL4 is negatively correlated with low-density lipoprotein (LDL) cholesterol and high-density lipoprotein (HDL) cholesterol and is positively correlated with various lipid metabolic syndromes [24]. The overexpression of ANGPTL4 increases plasma TG levels [25]. ANGPTL4 is also an endogenous inhibitor to intestinal fatty digestive enzymes. The knockout of ANGPTL4 in mice would increase the body weight, decreased the lipid content in feces, and increased dietary triglyceride accumulation in the small intestine, which was consistent with the increased intestinal lumen lipase activity $[21,26]$. ANGPTL3 mainly inhibits LPL activity in the feeding state, whereas ANGPTL4 plays an important role in both feeding and fasting states [1]. Different from ANGPTL3, ANGPTL4 is considered to be an endocrine or autocrine/paracrine inhibitor of LPL due to its different expression sites [27].

ANGPTL8 is a new protein mainly expressed in the human liver and plays an important role in regulation of plasma TG levels and lipid metabolism [28]. In addition, the N-terminal domain of ANGPTL8 is $20 \%$ identical to ANGPTL3 and ANGPTL4 and functionally binds to LPL to regulate triglyceride metabolism, indicating the affinity between them $[29,30]$. ANGPTL8 could interact with ANGPTL3, and they could synergistically inhibit LPL activity and regulate the plasma triglyceride levels [31, 32]. The overexpression of ANGPTL8 in the liver of ANGPTL3 knockout mice failed to increase the levels of triacylglycerol nonesterified fatty acids in plasma [33]. Since ANGPTL3, ANGPTL4, and ANGPTL8 are all LPL inhibitors, the absence of anyone of them would break the balance of triglyceride metabolism in turn would lead to hypotriglyceridemia or hypertriglyceridemia. During fed-fast cycle, feeding could induce the expression of ANGPTL8 and activate the interaction between ANGPTL8 and ANGPTL3 in mice, thereby inhibiting LPL activity in the myocardium and bones. Conversely, fasting could inhibit the expression of ANGPTL8 and improve the expression of ANGPTL4 in mice, resulting the transportation of TG to muscles [34].

So far, studies on the ANGPTLs have focused on mice [35] and humans [36], but reports on the ANGPTL gene in domestic animals are limited. The Jinhua pig is an important breed in China with a high percentage of body fat and good meat quality. Pigs are more closely related to humans in carbohydrate and lipid metabolism than mice [37]. Compared to mice, pigs are considered to be more suitable to be developed as animal models for studying human obesity and fat metabolism $[38,39]$. In this study, we reported the basic information of the ANGPTL1-8 gene in Jinhua pigs, the evolutionary relationship between the pig ANGPTL1-8 gene and other different vertebrates, their expression profile in different tissues, and the differences in the mRNA expression levels of ANGPTL3, ANGPTL4, and ANGPTL8 between 
obese and lean Jinhua pigs. The purpose of this study is to establish a theoretical foundation for the roles of the ANGPTL family genes in the fat regulation of pigs.

\section{Materials And Methods}

\section{Data Mining and Gene Identification}

The genome of pig ANGPTL family members was retrieved from the NCBI database to obtain basic information, such as transcript ID and mRNA length(bp) [40]. The relative molecular weights and charge was performed using the programs within the DNASTAR Lasergene [41], the ClustalW was used to generate the amino acid sequences [42]. Exon-intron boundaries and chromosomal locations were identified by the mRNA-genome alignment program Spidey [43].

\section{Phylogenetic and Evolutionary Analyses}

The MUSCLE program was used to align amino acid sequences (obtained from the NCBI database), and Boxhade software was used for visual display. Phylogenetic analysis of the ANGPTL family members in humans and animals, including mice, cattle, pigs, dogs, and chickens, was carried out using the deduced mature protein sequences. The Mega7.0 software was used to construct phylogenetic trees from P-distances of ANGPTL amino acid sequences from pigs and other representative vertebrates [44]. The Bootstrap method was used to test the reliability of each branch 1000 times.

\section{Short-range gene linkage}

In order to further confirm the homology of the ANGPTL gene and establish the evolutionary model of the ANGPTL gene in metazoa, we isolated the genetic environment of ANGPTL3 and ANGPTL4 chromosomes or genomic fragments of vertebrates to determine whether there are homologous genomic regions in vertebrates. Similarly, the genetic environment of mammalian ANGPTL8 is described. The short-range gene linkage comparison was performed among humans, mice, cattle, pigs, dogs, and poultry. The adjacent genetic environment of vertebrates was retrieved from the Genomicus database, and the genetic environment of human ANGPTL3, ANGPTL4, and ANGPTL8 were used as a reference sequence [45]. Homologous genomic regions were characterized by the discovery of specific genomic combinations of conserved flanking genes.

\section{Animals and Sampling}

A total of 60 Jinhua Pigs at 90-day old were obtained from the experimental pig farm of Jinhua Academy of Agricultural Sciences (Jinhua, Zhejiang Province, China). All Jinhua pigs were reared in pens (10 pens, 6 pigs per pen) in an environmentally controlled facility and had free access to commercial diets and water under a standard management. At 270 days of age, pigs were weighed invidually and slaughtered. Backfat thickness was measured. Based on the body weight data, different tissues were collected from 6 pigs with average body weight, immediately frozen in liquid nitrogen, and stored in $\mathrm{a}-80^{\circ} \mathrm{C}$ freezer until RNA isolation. The tissues included tongue, esophagus, stomach, duodenum, jejunum, ileum, cecum, colon, trachea, lung, heart, longissimus muscle, subcutaneous fat, liver, kidney, brain, pancreas, and spleen. Eight of $92.35 \pm 10.10 \mathrm{~kg}$ pigs with the higher and lower backfat thickness were set as the high group (H group) and low group ( $L$ group), respectively [46]. The ileum, subcutaneous fat, and liver segments of the $H$ and $L$ group pigs were collected, immediately frozen in liquid nitrogen, and stored in a $-80^{\circ} \mathrm{C}$ freezer until RNA isolation.

\section{RNA Extraction and Real-Time Quantitative PCR}

According to the manufacturer's instructions, total RNA was extracted from each tissue sample using a TRI-zol Plus RNA Purification Kit (Invitrogen, Carlsbad, CA). RNA integrity and purity were assessed by Nanodrop after being electrophoresed in a formaldehyde gel[47,48]. The genomic DNA contamination was removed from the RNA samples 
using a gDNA Eraser (Takara Bio Inc., Dalian, China), and the PrimeScript RT reagent kit (Takara Bio Inc.) was subsequently used according to the manufacturer's instructions for reverse transcription. The cDNA was then diluted ten times with RNase-free water before RT-qPCR analysis. RT-qPCR was performed on each sample by using the CFX96 RTqPCR Detection System (Bio-Rad Laboratories, Richmond, VA), and the amplification was conducted in a total volume of $20 \mu \mathrm{l}$, containing $10 \mu \mathrm{l}$ of SYBR Premix Ex Taq II (Takara Bio Inc.), $7 \mu \mathrm{l}$ of RNase-free water, $1 \mu$ l of the diluted cDNA, and $0.5 \mu$ of each primer (Table 1). The optimum RT-qPCR program was $95^{\circ} \mathrm{C}$ for $1 \mathrm{~min}$, followed by 40 cycles of $95^{\circ} \mathrm{C}$ for $15 \mathrm{~s}$ and $60^{\circ} \mathrm{C}$ for $25 \mathrm{~s}$. Relative quantification of the mRNA transcripts was accomplished using the $2^{-\triangle \triangle C T}$ method and the housekeeping gene was glyceraldehyde-3-phosphate dehydrogenase (GAPDH).

Table 1

Primers sequences used in the RT-qPCR analysis.

\begin{tabular}{|c|c|c|c|}
\hline Gene & Genbank Accession & Primer Sequences(5'to3') & Size(bp) \\
\hline \multirow[t]{2}{*}{ Pig ANGPTL1 } & \multirow[t]{2}{*}{ NM_001109947.1 } & GGCAACTCCTCCCACCAAA & \multirow[t]{2}{*}{106} \\
\hline & & GACTGAATGTCCAGCTTCTTTTGCA & \\
\hline \multirow[t]{2}{*}{ Pig ANGPTL2 } & \multirow[t]{2}{*}{ NM_001109946.1 } & GGCTCCGTCAACTTCTTTAGG & \multirow[t]{2}{*}{123} \\
\hline & & CAGTTTGTAGTTGCCTTGGTTCGT & \\
\hline \multirow[t]{2}{*}{ Pig ANGPTL3 } & \multirow[t]{2}{*}{ NM_001003926.2 } & GCACTCCCAGAACACGAAGA & \multirow[t]{2}{*}{96} \\
\hline & & CCACCAGCCTCCTGAATAACT & \\
\hline \multirow[t]{2}{*}{ Pig ANGPTL4 } & \multirow[t]{2}{*}{ NM_001038644.1 } & GACTGCCAAGAGCTGTTTGAAGA & \multirow[t]{2}{*}{126} \\
\hline & & CTGAATTACAGTCCAGCCTCCAT & \\
\hline \multirow[t]{2}{*}{ Pig ANGPTL5 } & \multirow[t]{2}{*}{ XM_003129804.4 } & CCAAAGGCTGTGGTGTGATTATC & \multirow[t]{2}{*}{140} \\
\hline & & CATCTTCAGATTCCAGGGCTACA & \\
\hline \multirow[t]{2}{*}{ Pig ANGPTL6 } & \multirow[t]{2}{*}{ XM_003123226.5 } & GTATCAGCATGGTGCGAGCAA & \multirow[t]{2}{*}{99} \\
\hline & & GTGCTGCCAAGTAGTGAAGAAGT & \\
\hline \multirow[t]{2}{*}{ Pig ANGPTL7 } & \multirow[t]{2}{*}{ NM_001142828.2 } & CTGGAGGTTTTCTGCGACATG & \multirow[t]{2}{*}{116} \\
\hline & & CCGAAGCCTTGCTTGTACTGT & \\
\hline \multirow[t]{2}{*}{ Pig ANGPTL8 } & \multirow[t]{2}{*}{ XM_021083871.1 } & GGACCTGTCACGCACCAA & \multirow[t]{2}{*}{78} \\
\hline & & CCGATCCCAGCCAACAG & \\
\hline \multirow[t]{2}{*}{ Pig GAPDH } & \multirow[t]{2}{*}{ NM_001206359.1 } & CCAGGGCTGCTTTTAACTCTG & \multirow[t]{2}{*}{104} \\
\hline & & GTGGGTGGAATCATACTGGAACAT & \\
\hline
\end{tabular}

\section{Statistical Analysis of Data}

Data were performed by SPSS 23.0 (IBM, New York, NY, United States) followed by an unpaired two-tailed Student's t-test (49). Relative expression data are presented as mean \pm standard error of the mean (SEM). Statistical significance was considered at $P<0.05$. The relative expression levels of ANGPTL3, ANGPTL4, and ANGPTL8 in different tissues from Jinhua Pigs with different amounts of body fat were performed with the GraphPad Prism version 8.00 (GraphPad Software, LaJolla California USA).

\section{Results}




\section{Basic Information of Pig ANGPTL1-8}

The nucleotide sequences of pig ANGPTL1-8 were obtained from the NCBI database, and the basic information was generated by DNASTAR Lasergene (Table 1). The mRNA sequences of pig ANGPTL1-8 were between 1100 and 2000 bp in length; the numbers of amino acid residues of ANGPTL1-7 were between 344-493 while ANGPTL8 was 198. ANGPTL1-8 was composed of 4-9 exons determined by Spidy program analysis (Table 1).

Table 2

Basic Information of Pig ANGPTL1-8

\begin{tabular}{|c|c|c|c|c|c|c|c|c|}
\hline Name & Transcript ID & $\begin{array}{l}\text { mRNA } \\
\text { length } \\
\text { (bp) }\end{array}$ & $\begin{array}{l}\text { Protein } \\
\text { (aa) }\end{array}$ & $\begin{array}{l}\text { relative } \\
\text { molecular } \\
\text { mass }\end{array}$ & $\begin{array}{l}\text { Exon } \\
\text { no. }\end{array}$ & Chromosome & $\begin{array}{l}\text { Negatively } \\
\text { charged } \\
\text { residues } \\
\text { (Asp + } \\
\text { Glu) }\end{array}$ & $\begin{array}{l}\text { Positively } \\
\text { charged } \\
\text { residues } \\
\text { (Arg + } \\
\text { Lys) }\end{array}$ \\
\hline ANGPTL1 & NM_001109947.1 & 1856 & 491 & 56582.68 & 4 & 9 & 57 & 61 \\
\hline ANGPTL2 & NM_001109946.1 & 1991 & 493 & 56952.39 & 4 & 1 & 55 & 56 \\
\hline ANGPTL3 & NM_001003926.2 & 1453 & 462 & 53658.25 & 7 & 6 & 67 & 54 \\
\hline ANGPTL4 & NM_001038644.1 & 1833 & 412 & 45545.53 & 7 & 2 & 39 & 47 \\
\hline ANGPTL5 & XM_003129804.4 & 1747 & 388 & 44536.36 & 8 & 9 & 48 & 42 \\
\hline ANGPTL6 & XM_003123226.5 & 1849 & 463 & 50726.18 & 5 & 2 & 43 & 53 \\
\hline ANGPTL7 & NM_001142828.2 & 1186 & 344 & 39394.58 & 6 & 6 & 41 & 44 \\
\hline ANGPTL8 & XM_021083872.1 & 1884 & 198 & 21979.25 & 4 & 2 & 19 & 16 \\
\hline
\end{tabular}

\section{Sequence Conservation of Pig ANGPTLs}

Multiple sequence alignment of amino acid sequences of pig ANGPTL1-8 showed that the amino acid sequence of ANGPTL1-7 was highly conserved, and the amino acid sequence of ANGPTL8 was less conserved (Fig. 1). Therefore, the structure of ANGPTL8 may be different from other members in the ANGPTL family. According to the Editsequence calculation, amino acid sequences of pig ANGPTL1-8 had 95.93\%, 97.57\%, 84.13\%, 79.37\%, 91.75\%, 88.35\%, 89.28\%, and $71.72 \%$ homology with the corresponding amino acid sequences of human ANGPTL1-8.

\section{Phylogeny of the Pig ANGPTLs}

Phylogenetic analysis of the vertebrate ANGPTL family suggested that these genes shared a common origin, and the family members emerged early during metazoan radiation (Fig. 2). According to the dendritic topology, there are five main protein clusters that contain different members of the ANGPTL family: the ANGPTL1-2-6 cluster (ANGPTL1, ANGPTL2, ANGPTL6), the ANGPTL3-4 cluster (ANGPTL3, ANGPTL4), the ANGPTL5 cluster, the ANGPTL7 cluster, and the ANGPTL8 cluster, which was isolated from the other four protein clusters (Fig. 2). Each ANGPTL orthologous gene was clustered with other mammalian sequences and separately from poultry.

\section{Neighbouring Gene Analysis}

By analyzing and comparing the genetic environment of ANGPTL3 (Fig. 3) and ANGPTL4 (Fig. 4) in mammals and poultry, we could further understand the evolutionary relationship of ANGPTL family genes in vertebrate radiation. The 
pig ANGPTL3 gene was mapped to chromosome 6, and the ANGPTL4 gene was mapped to chromosome 2 and was also found to be present in other mammals and chickens. Among the ten genes flanking human ANGPTL3, nine were retained on pig chromosome 6, and seven were retained on chicken chromosome 8. Of the ten genes flanking human ANGPTL4, nine were retained on pig chromosome 2 , and nine were retained on chicken chromosome 28 . This indicated that the genetic environment of several major mammals (humans, mice, cattle, pigs, and dogs) ANGPTL3 was highly similar and was also similar to that of chicken ANGPTL3, indicating that mammals and poultry ANGPTL3 and ANGPTL4 shared a common ancestral origin. Comparing the adjacent genetic environment of mammalian ANGPTL8 (Fig. 5) in pigs, ANGPTL8 was located on chromosome 2. Among the ten genes flanking human ANGPTL8, all were retained on cow chromosome 7, and nine genes were retained on pig chromosome 2. This indicated that the genetic environment of ANGPTL8 in several major mammals (humans, mice, cattle, pigs, dogs) were highly similar, which means that the mammalian ANGPTL8 had a common ancestral origin.

\section{Gene expression pattern of pig ANGPTL family}

In order to study the expression patterns of the ANGPTL gene family in pigs, the mRNA abundance of ANGPTL1-8 in 18 tissues of 6 Jinhua pigs was determined by RT-qPCR. As shown in Fig. 6, ANGPTL4 was widely expressed in various tissues, while ANGPTL3 and ANGPTL8 were predominantly expressed in the liver, and ANGPTL8 was only expressed in the liver. Meanwhile, it was observed that ANGPTL4 was highly expressed in the intestinal tissues with relative low expression in subcutaneous fat, lung, heart, and longissimus muscle. ANGPTL4 relative expression level was the highest in subcutaneous fat.

\section{Expression levels of ANGPTL3, ANGPTL4 and ANGPTL8 in Jinhua pigs with distinct fatness}

To confirm whether lipid metabolism was associated with the expression of ANGPTL3, ANGPTL4, and ANGPTL8, we selected six Jinhua pigs with average body weight for the determination of body weight, backfat thickness, and expression level of ANGPTL3, ANGPTL4, and ANGPTL8 by RT-qPCR analysis following RNA isolation and reverse transcription. The backfat thickness of Jinhua pigs in the $\mathrm{H}$ group was significantly higher than that of Jinhua pigs in the $\mathrm{L}$ group (Fig. 7). However, there were no significant differences in the body weight of Jinhua pigs between the $\mathrm{H}$ and $\mathrm{L}$ groups (Fig. 7). The relative expression of ANGPTL3 in the liver of the $\mathrm{H}$ group pigs was significantly lower than that of the $\mathrm{L}$ group pigs $(P=0.005)$ while the relative expression levels of ANGPTL4 in the ileum $(P=0.043)$, subcutaneous fat $(P=0.025)$, and liver $(P=0.008)$ of the $\mathrm{H}$ group pigs were lower than those of the $\mathrm{L}$ group pigs (Fig. 8). However, the relative expression of ANGPTL8 in the liver of the $\mathrm{H}$ group pigs was not significantly different from that of the $L$ group pigs $(P>0.05)$.

\section{Discussion}

The Jinhua pig has become one of the most important local breeds in China due to its excellent meat quality and it exhibits the characteristics of early sexual maturity, low prolificacy, and a high body fat content [50]. The purpose of this study was to explore the evolutionary relationship of ANGPTL family genes in Jinhua Pig, a model animal of lipid metabolism, and the important roles of ANGPTL3, ANGPTL4 and angptl8 in lipid metabolism. In the present study, amino acid sequence analysis, phylogenetic analysis, chromosome adjacent gene analysis, and RT-qPCR analysis showed that Jinhua pig ANGPTL family members had common characteristics with homologous human genes, including the high similarity of amino acid sequence and chromosome flanking genes. Consistent with earlier studies [51, 52], pig ANGPTL3-4 had typical angiopoietin family structures. The structures of pig ANGPTL3-4 was similar to those of humans and mice, and both had FReD, which affects angiogenic activity, and CCD, which regulated fat metabolism by inhibiting LPL. The eight ANGPTL family member genes in Jinhua pigs were 1100-2000 bp in length and consisted of 4-9 exons. The functional helical domain and fibrinogen-like domain in the ANGPTL1-8 amino acid sequence were strongly 
conserved. Through the identification and expression analysis of the bovine ANGPTL1-7 genes, all of the deduced amino acid sequences had an $\mathrm{N}$-terminal crimped domain and a C-terminal fibrinogen-like domain, and both had the characteristics of angiogenin. The bovine ANGPTL1-7 amino acid sequences are similar to those of humans, and the corresponding members had 95\%, 97\%, 82\%, 85\%, 87\%, 85\%, and $87 \%$ homology [43]. Related studies reported the cloning, chromosomal location, and expression analysis of pig ANGPTL3 and ANGPTL4. The deduced amino acid sequence of pig ANGPTL3 had $83 \%$ and $73.7 \%$ homology to humans and mice, respectively. While pig ANGPTL4 had $79.4 \%$ and $79.1 \%$ homology with humans and mice [52]. This research showed that pig ANGPTL1-8 amino acid sequence had $95.93 \%, 97.57 \%, 84.13 \%, 79.37 \%, 91.75 \%, 88.35 \%$, and $89.28 \%$ homology with corresponding human members, respectively.

In vertebrates, the ANGPTL family of genes evolved by gene duplication, gene deletion, and gene mutation. They share the same evolutionary origin with angiogenin and share similarities in sequence and structure. ANGPTL family members expanded in early vertebrate genome doubling and genome segment replication before vertebrate radiation. Five major ANGPTL vertebrate protein clusters, ANGPTL1-2-6, ANGPTL3-4, ANGPTL5, ANGPTL7, ANGPTL8, derived from the replication of ancestor ANGPTL $[53,54]$. The ANGPTL8 cluster was isolated from the other four protein clusters since ANGPTL8 has a different structure from other ANGPTL family members [33]. Clustering in phylogenetic trees indicates that pig sequences are not significantly different from those of other vertebrates.

Recent studies have shown that ANGPTL3 [55], ANGPTL4 [56], and ANGPTL8 [57] are directly related to lipid metabolism and fat deposition in the body. In this study, tissue expression profiles showed that ANGPTL3 and ANGPTL8 were mainly expressed in the liver of pigs, with similar results in humans and mice $[58,59]$. However, ANGPTL4 in pigs is widely expressed in many tissues, including the gastrointestinal tract, subcutaneous fat, liver, muscles, heart, and lungs. In humans and mice, ANGPTL4 is also expressed mainly in white adipose tissue and the liver as well as in the intestine and heart tissue $[18,60]$. By analyzing the expression levels of ANGPTL3 and ANGPTL8 in the liver and the relative expression levels of ANGPTL4 in the liver, subcutaneous fat, and ileum of Jinhua pigs with distinct fatness, we found that the expression of ANGPTL3 in the liver of Jinhua pigs in the $\mathrm{H}$ group was significantly lower than that of Jinhua pigs in the $\mathrm{L}$ group $(P=0.005)$. Moreover, the expression level of ANGPTL4 in the liver $(P=0.008)$, subcutaneous fat $(P=$ $0.025)$, and ileum $(P=0.043)$ of the $\mathrm{H}$ group pigs was significantly lower than that of the $\mathrm{L}$ group pigs. This might be due to the inhibitory effect of ANGPTL3 and ANGPTL4 on LPL. With the decrease in the expressions of ANGPTL3 and ANGPTL4, the activity of LPL would increase, which in turn would decrease the serum TG level and increase the lipid absorption. Early research showed that in sterile mice with routine feeding, the microbiota promoted the storage of triglycerides in fat cells by inhibiting the intestinal expression of ANGPTL4, illustrating the decrease of ANGPTL4 expression is beneficial to fat storage [20]. Recent studies have indicated that the level of circulating ANGPTL4 in obese children was lower than that of normal weight children. Waist circumference, body weight, and other major obesity indicators were negatively correlated with the level of circulating ANGPTL4 [61]. Meanwhile, mice with ANGPTL3 and ANGPTL4 deficiency had severe hypertriglyceridemia while mice with ANGPTL3 overexpression had hyperlipidemia [62, 63]. Furthermore, compared with other breeds of pigs, the fat percentage of Large White pigs was lower with a higher expression of ANGPTL4 mRNA [64]. These studies are consistent with the results of the present study.

Although there was no significant difference in ANGPTL8 expression between the liver of Jinhua pigs in the $\mathrm{H}$ and $\mathrm{L}$ groups, it might be resulted from the function of ANGPTL8, which promotes the cleavage of ANGPTL3 and binds to the $\mathrm{N}$-terminal of ANGPTL3 [34]. The interaction of ANGPTL8 with ANGPTL3 would form a complex with the N-terminal of ANGPTL3, synergistically inhibiting the LPL activity and modulating the plasma triglyceride levels [31]. Additionally, the overexpression of ANGPTL8 in the liver of ANPTL3 gene knockout mice had no effect on the triglyceride metabolism [33].

Fat metabolism and faat storage are closely related to the meat quality and carcass quality. Relevant research showed that by comparing the carcass composition and development patterns of Jinhua and Landrace pigs at $35 \sim 125$ days of

Page $8 / 21$ 
age, the carcass fat content of Jinhua pigs was significantly higher than that of Landrace pigs $(P<0.05)$, and the lean meat rate of the carcass was significantly lower than that of Landrace pigs $(P<0.01)$ [65]. Similar studies showed that Jinhua pigs had a higher tendency to deposit fat compared with Landrace pigs[39]. These are consistent with the results of this experiment. Therefore, Jinhua Pigs with high body fat percentage and good meat quality play an important role in the study of lipid metabolism and carcass quality.

\section{Conclusion}

Collectively, the present study identified ANGPTL family members in Jinhua pigs. The expression of ANGPTLs was tissue-specific in Jinhua pigs. Moreover, the expression levels of ANGPTL3 and ANGPTL4 were associated with fat metabolism in Jinhua pigs. This study would enrich our knowledge of the pig ANGPTL gene family and confirm the importance of ANGPTL3 and ANGPTL4 in regulating the lipid metabolism and improving the carcass quality of pigs.

\section{Abbreviations}

ALG6 ALG6 alpha-1,3-glucosyltransferase

ANG Angiopoietins

ANGPTL Angiopoietin-like

ANGPTL3 Angiopoietin-like 3

ANGPTL4 Angiopoietin-like 4

ANGPTL8 Angiopoietin-like 8

ATG4C Autophagy related 4C cysteine peptidase

CCD Coiled-coil domain

CCDC159 Coiled-coil domain containing 159

CD320 CD320 molecule

CERS 4 ceramide synthase 4

DOCK6 Dedicator of cytokinesis 6

EFCAB7 EF-hand calcium binding domain 7

FIAF Fasting-induced adipokine

FOXD3 Forkhead box D3

FReD Fibrinogen domain

GAPDH Glyceraldehyde-3-phosphate dehydrogenase

HNRNPM Heterogeneous nuclear ribonucleoprotein M

HDL High-density lipoprotein 
ITGB3BP Integrin subunit beta 3 binding protein

KANK2 KN motif and ankyrin repeat domains 2

KANK3 KN motif and ankyrin repeat domains 3

KANK4 KN motif and ankyrin repeat domains 4

L1TD1 LINE1 type transposase domain containing 1

LDL Low-density lipoprotein

LDLR Low density lipoprotein receptor

LPL Lipoprotein lipase

LSD Least Significant Difference

LXR Liver X receptor

MARCH2 Membrane-associated ring finger (C3HC4) 2

NDUFA7 NADH:ubiquinone oxidoreductase subunit A7

PAJT PATJ crumbs cell polarity complex component

PGAR Peroxisome proliferator-activated receptor gamma angiopoietin-related protein

PLPPR2 Phospholipid phosphatase related 2

PRAM1 PML-RARA regulated adaptor molecule 1

RAB3D RAB3D, member RAS oncogene family

Rab11B RAB11B, member RAS oncogene family

RPS28 Ribosomal protein S28

SMARCA4 SWI/SNF related, matrix associated, actin-dependent regulator of chromatin, subfamily a, member 4 SPAN16 Sperm peptide antigen

SPC24 SPC24 component of NDC80 kinetochore complex

SS Signal sequence

TG Triglyceride

TMEM250 Transmembrane protein 250

USP1 Ubiquitin-specific peptidase 1

VLDL very low density lipoprotein

WAT White adipose tissue 


\section{Declarations}

\section{ACKNOWLEDGMENTS}

The authors would like to express their thanks to the personnel of these teams for their selfless assistance.

\section{Authors' contributions}

$Z Z, W L, Y X$, and RY designed the experiment. ZB, WL, QH, HY, YR, and YX conducted the animal experiments. ZZ, WL, HY, $R Y$, and $Y X$ wrote and revised the manuscript. $Z Z, W L, Y R, H Y$, and $Y X$ did the experimental analysis, collected, and analyzed the data. All authors reviewed the manuscript.

\section{Funding}

This study was funded by the National Natural Science Foundation of China (31972999) and the State Key Laboratory for Managing Biotic and Chemical Threats to the Quality and Safety of Agroproducts (2010DS700124-ZZ1905).

\section{Availability of data and materials}

The datasets reported in this manuscript are available from the corresponding author on reasonable request.

\section{Ethics approval and consent to participate}

All animals used in this study were reviewed and approved by the Institutional Animal Care and Use Committee of Zhejiang Academy of Agricultural Sciences. Written informed consent was obtained from the owners for the participation of their animals in this study.

\section{Consent for publication}

The authors declare consent for publication.

\section{Competing interests}

The authors declare no competing interests.

\section{References}

1. Dijk W, Kersten S. Regulation of lipid metabolism by angiopoietin-like proteins. Curr Opin Lipidol. 2016;27(3):24956.

2. Zhang R, Abou-Samra AB. Emerging roles of Lipasin as a critical lipid regulator. Biochem Biophys Res Commun. 2013;432(3):401-5.

3. Fu W, Ma Y, Chen N, Li H, Bai Y. [Expression of angiopoietin-like proteins for animal breeding: a review]. Sheng Wu Gong Cheng Xue Bao. 2015;31(11):1567-78.

4. Mengdie L, Daoquan P. ANGPTL8. An Important Regulator in Metabolic Disorders. Front Endocrinol (Lausanne). 2018;9:169.

5. Morelli MB, Chavez C, Santulli G. Angiopoietin-like proteins as therapeutic targets for cardiovascular disease: focus on lipid disorders. Expert Opin Ther Targets. 2020;24(1):79-88.

6. Ghaben AL, Scherer PE. Adipogenesis and metabolic health. Nat Rev Mol Cell Biol. 2019;20(4):242-58. 
7. Aron-Wisnewsky J, Warmbrunn MV, Nieuwdorp M, Clément K. Metabolism and Metabolic Disorders and the Microbiome: The Intestinal Microbiota Associated With Obesity, Lipid Metabolism, and Metabolic HealthPathophysiology and Therapeutic Strategies. Gastroenterology. 2021;160(2):573-99.

8. Kersten S. Angiopoietin-like 3 in lipoprotein metabolism. Nat Rev Endocrinol. 2017;13(12):731-9.

9. Li J, Li L, Guo D, Li S, Zeng Y, Liu C, et al. Triglyceride metabolism and angiopoietin-like proteins in lipoprotein lipase regulation. Clin Chim Acta. 2020;503:19-34.

10. Korn ED. Clearing factor, a heparin-activated lipoprotein lipase. I. Isolation and characterization of the enzyme from normal rat heart. J Biol Chem. 1955;215(1):1-14.

11. Davies BS, Beigneux AP, Barnes RH, Tu Y, Gin P, Weinstein MM, et al. GPIHBP1 is responsible for the entry of lipoprotein lipase into capillaries. Cell Metab. 2010;12(1):42-52.

12. Kersten S. Physiological regulation of lipoprotein lipase. Biochim Biophys Acta. 2014;1841(7):919-33.

13. Lichtenstein L, Kersten S. Modulation of plasma TG lipolysis by Angiopoietin-like proteins and GPIHBP1. Biochim Biophys Acta. 2010;1801(4):415-20.

14. Arca M, Minicocci I, Maranghi M. The angiopoietin-like protein 3: a hepatokine with expanding role in metabolism. Curr Opin Lipidol. 2013;24(4):313-20.

15. Kaplan R. Regulation of the angiopoietin-like protein 3 gene by LXR. J Lipid Res. 2003;44(1):136-43.

16. Hassan M. ANGPLT3: A novel modulator of lipid metabolism. Glob Cardiol sci Pract. 2017;2017(1):e201706.

17. Wang Y, Mcnutt MC, Banfi S, Levin MG, Holland WL, Gusarova V, et al. Proc Natl Acad Sci U S A. 2015;112(37):11630-35.

18. Ruppert PMM, Michielsen C, Hazebroek EJ, Pirayesh A, Olivecrona G, Afman LA, et al. Fasting induces ANGPTL4 and reduces LPL activity in human adipose tissue. Mol Metab. 2020;40:101033.

19. Cushing EM, Chi X, Sylvers KL, Shetty SK, Potthoff MJ, Davies BSJ. Angiopoietin-like 4 Directs Uptake of Dietary Fat Away From Adipose During Fasting. Mol Metab. 2017;6(8):809-18.

20. Bäckhed F, Ding H, Wang T, Hooper LV, Koh GY, Nagy A, et al. The gut microbiota as an environmental factor that regulates fat storage. Proc Natl Acad Sci U S A. 2004;101(44):15718-23.

21. Mattijssen F, Alex S, Swarts HJ, Groen AK, Van Schothorst EM, Kersten S. Angptl4 serves as an endogenous inhibitor of intestinal lipid digestion. Mol Metab. 2014;3(2):135-44.

22. Alex S, Lichtenstein L, Dijk W, Mensink RP, Tan NS, Kersten S. ANGPTL4 is produced by entero-endocrine cells in the human intestinal tract. Histochem Cell Biol. 2014;141(4):383-91.

23. Mcqueen AE, Kanamaluru D, Yan K, Gray NE, Wu L, Li ML, et al. The C-terminal fibrinogen-like domain of angiopoietin-like 4 stimulates adipose tissue lipolysis and promotes energy expenditure. J Biol Chem. 2017;292(39):16122.

24. Mehta N, Qamar A, Qu L, Qasim AN, Mehta NN, Reilly MP, et al. Differential association of plasma angiopoietin-like proteins 3 and 4 with lipid and metabolic traits. Arterioscler Thromb Vasc Biol. 2014;34(5):1057-63.

25. Yu X, Burgess SC, Ge H, Wong KK, Nassem RH, Garry DJ, et al. Inhibition of cardiac lipoprotein utilization by transgenic overexpression of Angptl4 in the heart. Proc Natl Acad Sci U S A. 2005;102(5):1767-72.

26. Lichtenstein L, Mattijssen F, de Wit NJ, Georgiadi A, Hooiveld GJ, van der Meer R, et al. Angptl4 protects against severe proinflammatory effects of saturated fat by inhibiting fatty acid uptake into mesenteric lymph node macrophages. Cell Metab. 2010;12(6):580-92.

27. Li C. Genetics and regulation of angiopoietin-like proteins 3 and 4. Curr Opin Lipidol. 2006;17(2):152-6.

28. Chen YQ, Pottanat TG, Siegel RW, Ehsani M, Qian YW, Roell WC, et al. Angiopoietin-like protein 4(E40K) and ANGPTL4/8 complex have reduced, temperature-dependent LPL-inhibitory activity compared to ANGPTL4. Biochem

Page $12 / 21$ 
Biophys Res Commun. 2021;534:498-503.

29. Ren G, Kim JY, Smas CM. Identification of RIFL, a novel adipocyte-enriched insulin target gene with a role in lipid metabolism. Am J Physiol Endocrinol Metab. 2012;303(3):E334-51.

30. Zhang R. Lipasin, a novel nutritionally-regulated liver-enriched factor that regulates serum triglyceride levels. Biochem Biophys Res Commun. 2012;424(4):786-92.

31. Chi X, Britt EC, Shows HW, Hjelmaas AJ, Shetty SK, Cushing EM, et al. Davies BSJ: ANGPTL8 promotes the ability of ANGPTL3 to bind and inhibit lipoprotein lipase. Mol Metab. 2017;6(10):1137-49.

32. Haller JF, Mintah IJ, Shihanian LM, Stevis P, Buckler D, Alexa-Braun CA, et al. ANGPTL8 requires ANGPTL3 to inhibit lipoprotein lipase and plasma triglyceride clearance. J lipid Res. 2017;58(6):1166-73.

33. Quagliarini F, Wang Y, Kozlitina J, Grishin NV, Hyde R, Boerwinkle E, et al. Atypical angiopoietin-like protein that regulates ANGPTL3. Proc Natl Acad Sci U S A. 2012;109(48):19751-6.

34. Zhang R. The ANGPTL3-4-8 model, a molecular mechanism for triglyceride trafficking. Open Biol. 2016;6(4):150272.

35. Graham MJ, Lee RG, Brandt TA, Tai LJ, Fu W, Peralta R, et al. Cardiovascular and Metabolic Effects of ANGPTL3 Antisense Oligonucleotides. N Engl J Med. 2017;377(3):222-32.

36. Dewey FE, Gusarova V, Dunbar RL, O'Dushlaine C, Schurmann C, Gottesman O, et al. Genetic and Pharmacologic Inactivation of ANGPTL3 and Cardiovascular Disease. N Engl J Med. 2017;377(3):211-21.

37. Poledne R, Jurčíková-Novotná L. Experimental models of hyperlipoproteinemia and atherosclerosis. Physiol Res. 2017;66(Suppl 1):69-75.

38. Gong Y, Zou X, Xia W, Wen X, Yang H. Comparative metabolomic analysis of caecal digesta between Jinhua pig and Landrace pig. Czech J Ani Sci. 2019;64(8):332-42.

39. Xiao Y, Kong F, Xiang Y, Zhou W, Wang J, Yang H, et al. Comparative biogeography of the gut microbiome between Jinhua and Landrace pigs. Sci Rep. 2018;8(1):5985.

40. Geer LY, Marchler-Bauer A, Geer RC, Han L, He J, He S, et al. The NCBI BioSystems database. Nucleic Acids Res. 2010;38(Database issue):D492-6.

41. Blanton LS, Quade BR, Bouyer DH. Differentiation of Rickettsia felis and Rickettsia felis-Like Organisms via Restriction Fragment Length Polymorphism Analysis. Vector Borne Zoonotic Dis. 2019;19(8):637-9.

42. Thompson JD, Higgins DG, Gibson TJ. CLUSTAL W: improving the sensitivity of progressive multiple sequence alignment through sequence weighting, position-specific gap penalties and weight matrix choice. Nucleic Acids Res. 1994;11(22):4673-80. 22(.

43. Zhengbing G, Guolin C, Junyong S, Jian L. Identification and expression analysis of bovine ANGPTL gene family. Curr Zool. 2010;56(4):445-53.

44. Kumar S, Stecher G, Tamura K. Molecular Evolutionary Genetics Analysis Version 7.0 for Bigger Datasets. Mol Biol Evol. 2016;33(7):1870-4.

45. Louis A, Nguyen NT, Muffato M, Roest Crollius H. Genomicus update 2015: KaryoView and MatrixView provide a genome-wide perspective to multispecies comparative genomics. Nucleic Acids Res. 2015;43(Database issue):D6829.

46. Yang H, Huang X, Fang S, Xin W, Huang L, Chen C. Uncovering the composition of microbial community structure and metagenomics among three gut locations in pigs with distinct fatness. Sci Rep. 2016;3:6:27427.

47. Jin L, Yu JP, Yang ZJ, Merilä J, Liao WB. Modulation of Gene Expression in Liver of Hibernating Asiatic Toads (Bufo gargarizans). Int J Mol Sci. 2018;19(8):2363.

48. Yan H, Zhou P, Zhang Y, Zhang Z, Zhang H. Short-chain fructo-oligosaccharides alleviates oxidized oil-induced intestinal dysfunction in piglets associated with the modulation of gut microbiota. J Funct Foods. 2020;64:103661.

Page 13/21 
49. Yang H, Xiang Y, Robinson K, Wang J, Zhang G, Zhao J, Xiao Y. Gut Microbiota Is a Major Contributor to Adiposity in Pigs. Front Microbiol. 2018;9:3045.

50. Yang $\mathrm{H}$, Xiao $Y$, Wang J, Xiang Y, Gong $Y$, Wen X, Li D. Core gut microbiota in Jinhua pigs and its correlation with strain, farm and weaning age. J Microbiol. 2018;56(5):346-55.

51. Ono M, Shimizugawa T, Shimamura M, Yoshida K, Nojisakikawa C, Ando Y, et al. Protein Region Important for Regulation of Lipid Metabolism in Angiopoietin-like 3 (ANGPTL3) ANGPTL3 is cleaved and activated in vivo. J Biol Chem. 2003;278(43):41804-9.

52. Feng SQ, Chen XD, Xia T, Gan L, Qiu H, Dai MH, et al. Cloning, chromosome mapping and expression characteristics of porcine ANGPTL3 and - 4. Cytogenet Genome Res. 2006;114(1):44-9.

53. Dehal P, Boore JL. Two rounds of whole genome duplication in the ancestral vertebrate. PLoS Bio. 2005;3(10):e314.

54. Asrar Z, Haq F, Abbasi AA. Fourfold paralogy regions on human HOX-bearing chromosomes: role of ancient segmental duplications in the evolution of vertebrate genome. Mol Phylogenet Evol. 2013;66(3):737-47.

55. Ruhanen H, Haridas PAN, Minicocci I, Taskinen JH, Palmas F, di Costanzo A, et al. ANGPTL3 deficiency alters the lipid profile and metabolism of cultured hepatocytes and human lipoproteins. Biochim Biophys Acta Mol Cell Biol Lipids. 2020;1865(7):158679.

56. Kristensen KK, Leth-Espensen KZ, Mertens HDT, Birrane G, Meiyappan M, Olivecrona G, et al. Unfolding of monomeric lipoprotein lipase by ANGPTL4: Insight into the regulation of plasma triglyceride metabolism. Proc Natl Acad Sci U S A. 2020;117(8):4337-46.

57. Kovrov O, Kristensen KK, Larsson E, Ploug M, Olivecrona G. On the mechanism of angiopoietin-like protein 8 for control of lipoprotein lipase activity. J Lipid Res. 2019;60(4):783-93.

58. Li X, Zhang Y, Zhang M, Wang Y. GALNT2 regulates ANGPTL3 cleavage in cells and in vivo of mice. Sci Rep. 2020;10(1):16168.

59. Catalano-Iniesta L, Sánchez Robledo V, Iglesias-Osma MC, Galán Albiñana A, Carrero S, Blanco EJ, et al. Evidences for Expression and Location of ANGPTL8 in Human Adipose Tissue. J Clin Med. 2020;9(2):512.

60. Santulli G. Angiopoietin-like proteins: a comprehensive look. Front Endocrinol (Lausanne). 2014;5:4.

61. Barja-Fernández S, Folgueira C, Castelao C, Pena-León V, González-Saenz P, Vázquez-Cobela R, et al. ANGPTL-4 is Associated with Obesity and Lipid Profile in Children and Adolescents. Nutrients. 2019;11(6):1340.

62. Köster A, Chao YB, Mosior M, Ford A, Gonzalez-DeWhitt PA, Hale JE, et al. Transgenic angiopoietin-like (angptl)4 overexpression and targeted disruption of angptl4 and angpt|3: regulation of triglyceride metabolism. Endocrinology. 2005;146(11):4943-50.

63. Xiao HB, Wang JY, Sun ZL. ANGPTL3 is part of the machinery causing dyslipidemia majorily via LPL inhibition in mastitis mice. Exp Mol Pathol. 2017;103(3):242-8.

64. Ren ZQ, Wu WJ, Liu WH, Zheng R, Li JL, Zuo B, et al. Differential expression and effect of the porcine ANGPTL4 gene on intramuscular fat. Genetics molecular research: Genet Mol Res. 2014;13(2):2949-58.

65. Miao ZG, Wang LJ, Xu ZR, Huang JF, Wang YR. Developmental changes of carcass composition, meat quality and organs in the Jinhua pig and Landrace. Animal. 2009;3(3):468-73.

\section{Figures}


ANGPTL1 ANGPTL2 ANGPTL 3 ANGPTL4 ANGPTL 5 ANGPTL 6 ANGPTL7 ANGPTL 8

ANGPTL1 ANGPTL2 ANGPTL 3 ANGPTL 4 ANGPTL5 ANGPTL 6 ANGPTL 7 ANGPTL8

ANGPTL1 ANGPTL2 ANGPTL 3 ANGPTL 4 ANGPTL 5 ANGPTL 6 ANGPTL7 ANGPTL 8

ANGPTL1 ANGPTL2 ANGPTL 3 ANGPTL 4 ANGPTL 5 ANGPTL 6 ANGPTL7 ANGPTL8

ANGPTL1 ANGPTL2 ANGPTL 3 ANGPTL 4 ANGPTL 5 ANGPTL6 ANGPTL 7 ANGPTL8
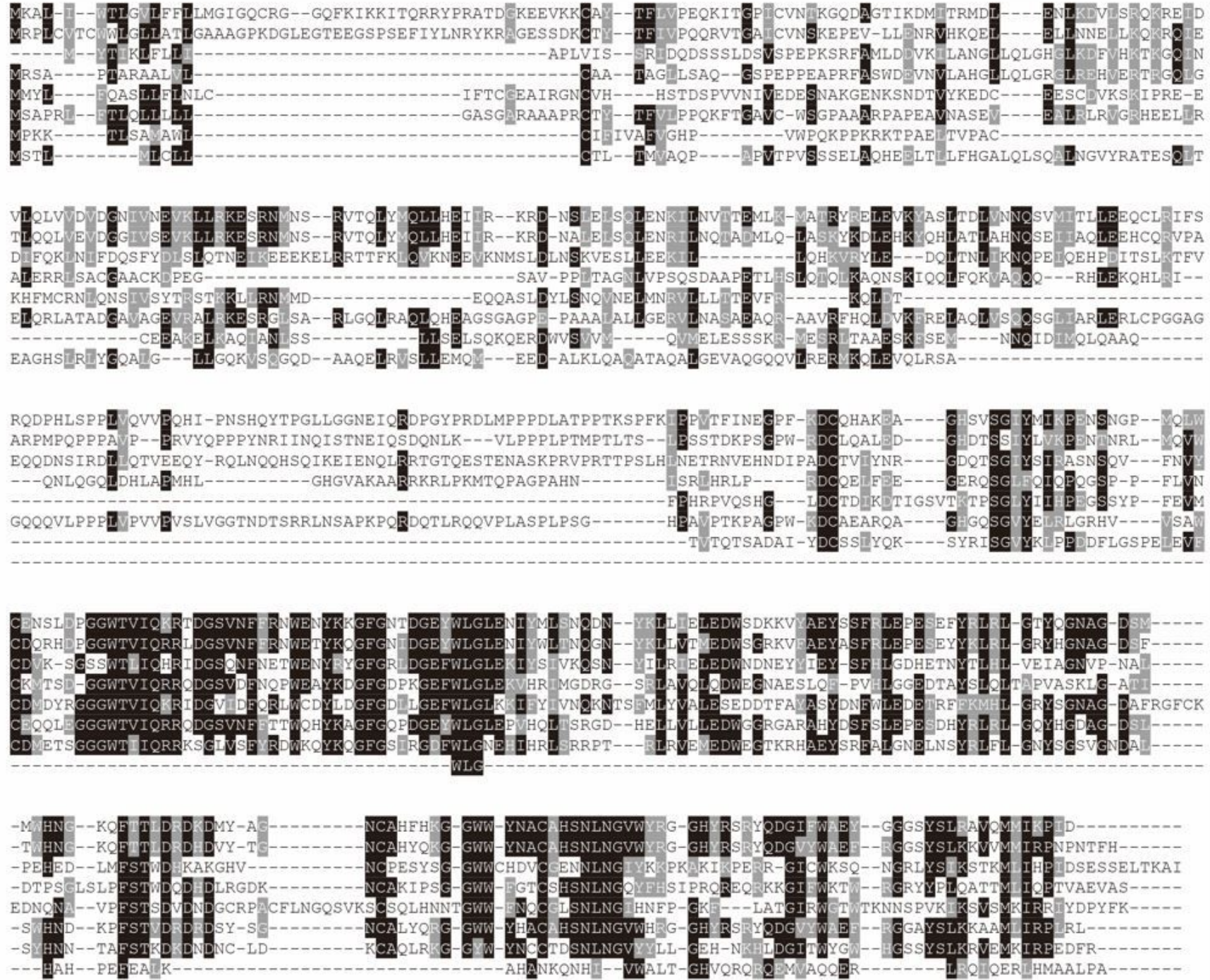

Figure 1

Multiple sequence alignment of representative pig ANGPTL protein sequences. The conserved residues are shaded. Specifically, the black part is highly similar, the gray part is less similar, and the non-color covered part has no similarity. 


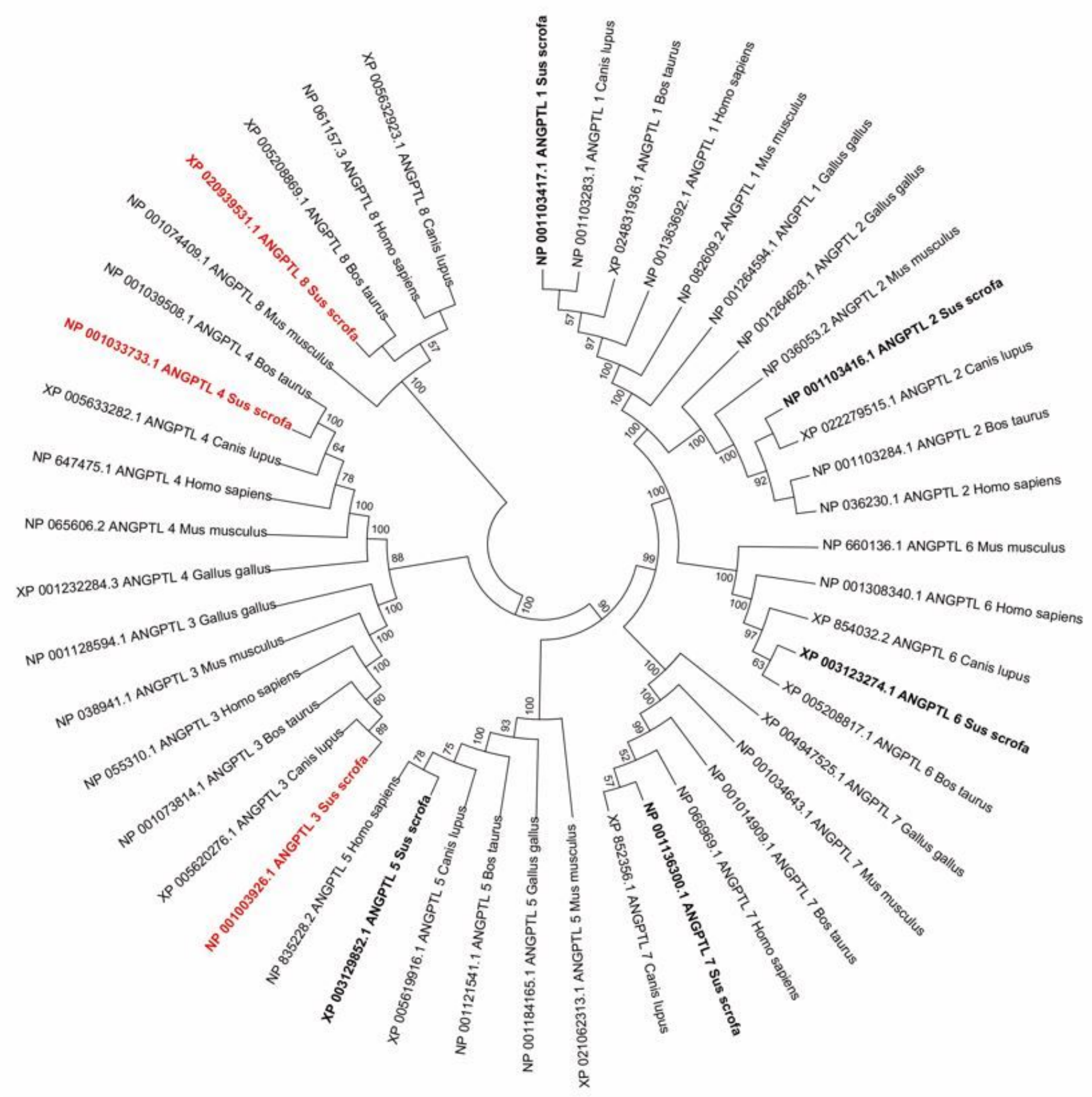

Figure 2

Phylogenetic analysis of pig ANGPTL genes and their representative sequences from human and animals. The topology was constructed using the amino acid sequences by the Neighbor-Joining method with 1000 bootstrap repeats. Only the branches with a bootstrap value greater than 50 are displayed at branching points. 


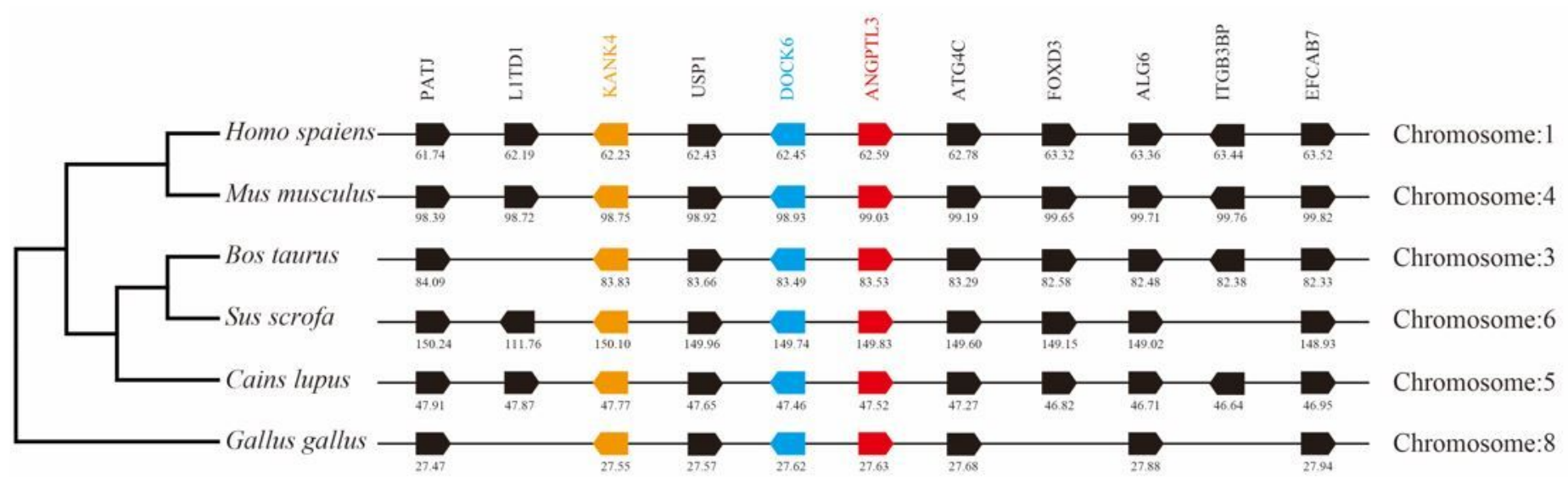

Figure 3

Comparison of homologous genomic regions of pig ANGPTL3 with humans, mice, and several other animals. The genetic environment of the pig ANGPTL3 gene was characterized, and the homologous genes of humans, mice, and other animals were identified. The horizontal line represents the chromosome fragments, the arrow box represents the gene, and the arrowhead points in the direction of predicted gene transcription. Only genes conserved across species are displayed. The same color is of the gene homologs, and they are presented according to their order in the chromosome. The gene names and symbols are: PATJ crumbs cell polarity complex component (PATJ), LINE1 type transposase domain containing 1 (L1TD1), KN motif and ankyrin repeat domains 4 (KANK4), ubiquitin-specific peptidase 1 (USP1), Dedicator of cytokinesis 6 (DOCK6), Angiopoietin-like 3 (ANGPTL3), Autophagy related 4C cysteine peptidase (ATG4C), Forkhead box D3 (FOXD3), ALG6 alpha-1,3-glucosyltransferase (ALG6), Integrin subunit beta 3 binding protein (ITGB3BP), EF-hand calcium binding domain 7 (EFCAB7).

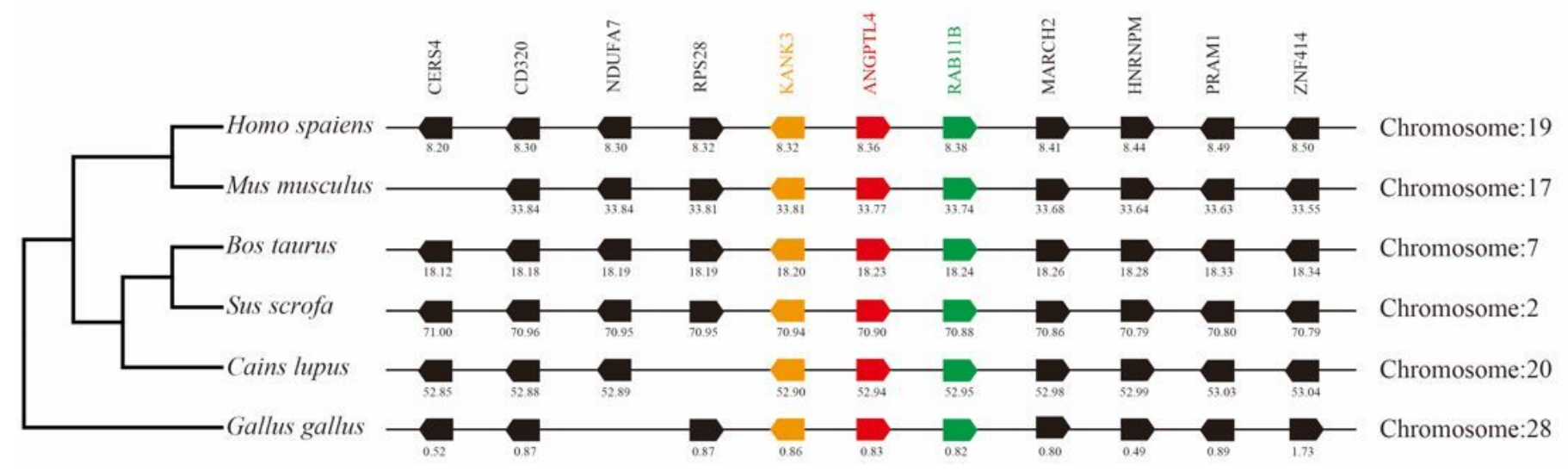

Figure 4

Comparison of homologous genomic regions of pig ANGPTL4 with humans, mice, and several other animals. The genetic environment of the pig ANGPTL4 gene was characterized, and the homologous genes of humans, mice, and other animals were identified. The horizontal line represents the chromosome segment, the arrow box represents the gene, and the arrowhead points in the direction of predicted gene transcription. Only genes preserved across species will appear. The same color is of the gene homologs, and they are presented according to their order in the chromosome. The gene names and symbols are: ceramide synthase 4 (CERS4), CD320 molecule (CD320), NADH:ubiquinone oxidoreductase subunit A7 (NDUFA7), Ribosomal protein S28 (RPS28), KN motif, and ankyrin repeat domains 3 (KANK3), 
Angiopoietin-like 4 (ANGPTL4), RAB11B, member RAS oncogene family (Rab11B), Membrane-associated ring finger (C3HC4) 2 (MARCH2), Heterogeneous nuclear ribonucleoprotein M (HNRNPM), PML-RARA regulated adaptor molecule 1 (PRAM1), Zinc finger protein 414 (ZNF414).

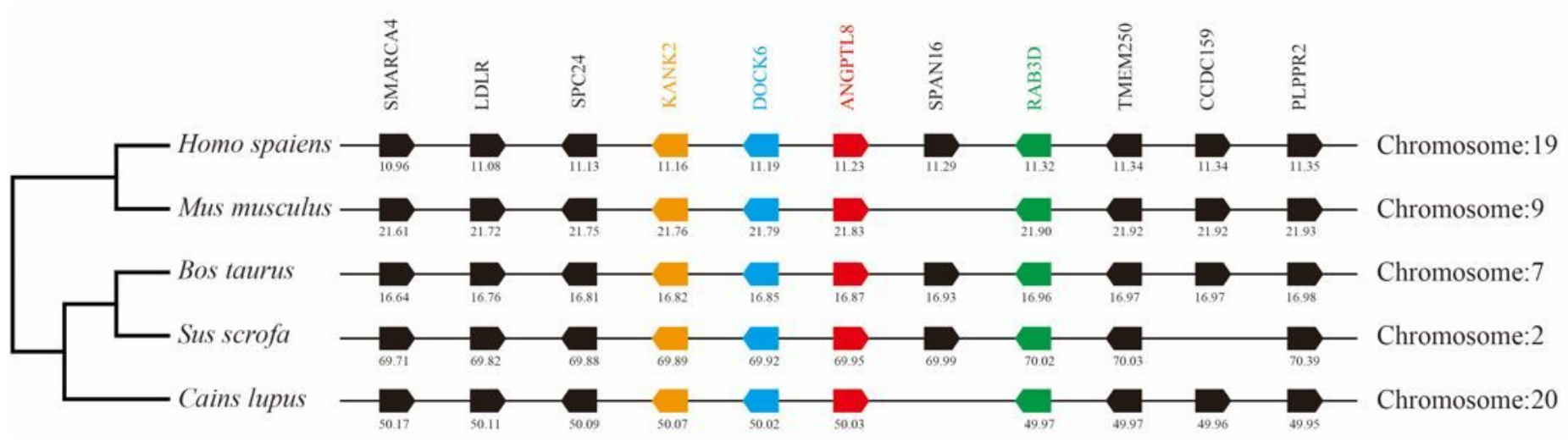

Figure 5

Comparison of homologous genomic regions of pig ANGPTL8 with humans, mice, and several other animals. The genetic environment of the pig ANGPTL8 gene was characterized, and the homologous genes of humans, mice, and other animals were identified. The horizontal line represents the chromosome segment, the arrow box represents the gene, and the arrowhead points in the direction of predicted gene transcription. Only genes preserved across species will appear. The same color is of the gene homologs, and they are presented according to their order in the chromosome. The gene names and symbols are: SWI/SNF related, matrix associated, actin-dependent regulator of chromatin, subfamily a, member 4 (SMARCA4), Low density lipoprotein receptor (LDLR), SPC24 component of NDC80 kinetochore complex (SPC24), KN motif and ankyrin repeat domains 2 (KANK2), Dedicator of cytokinesis 6 (DOCK6), Angiopoietin-like 8 (ANGPTL8), Sperm peptide antigen (SPAN16), RAB3D, member RAS oncogene family (RAB3D), Transmembrane protein 250 (TMEM250), Coiled-coil domain containing 159 (CCDC159), Phospholipid phosphatase related 2 (PLPPR2). 

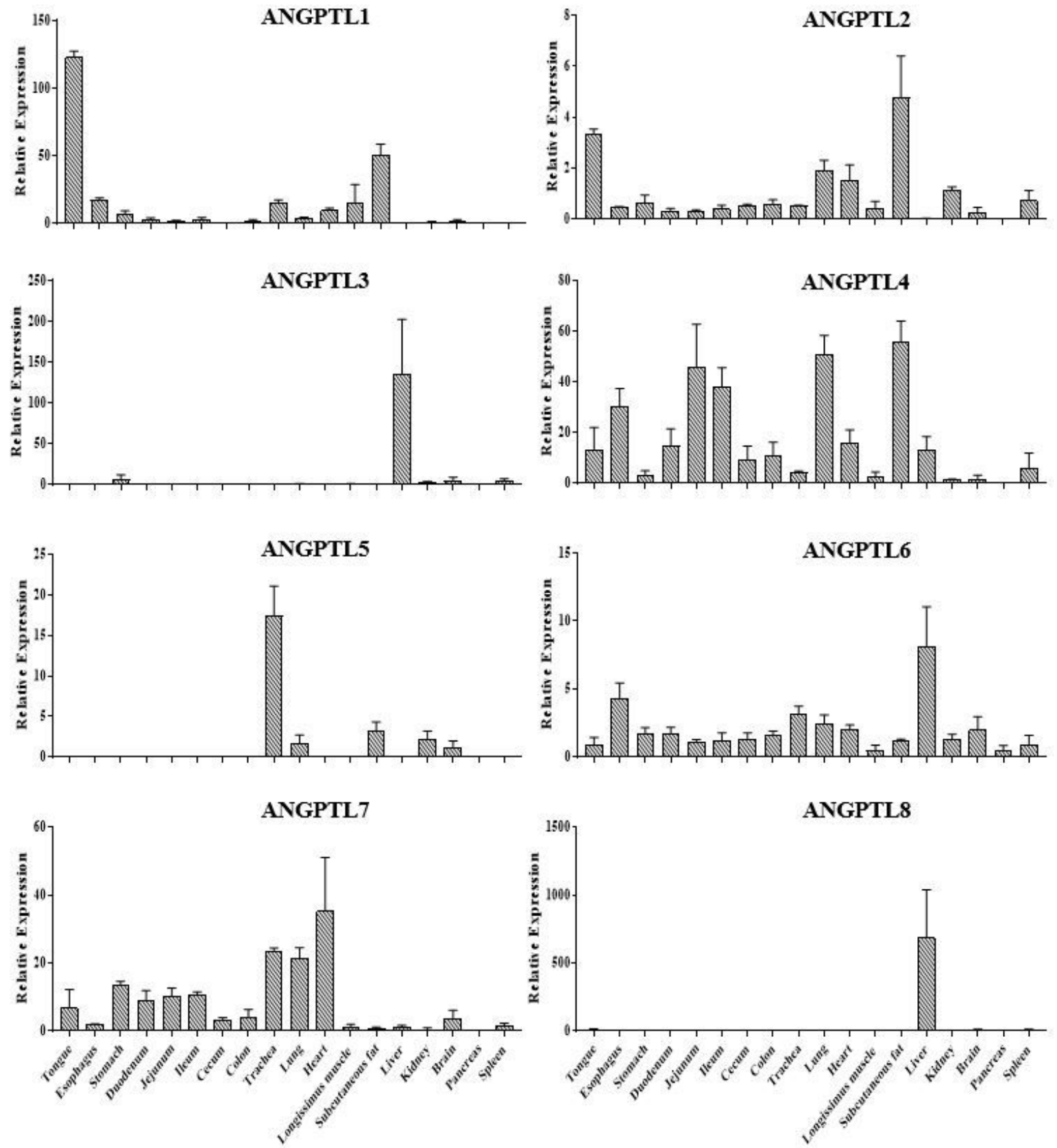

\section{Figure 6}

Relative expression of ANGPTL1-8 in different tissues, including tongue, esophagus, stomach, duodenum, jejunum, ileum, cecum, colon, trachea, lung, heart, longissimus muscle, subcutaneous fat, liver, kidney, brain, pancreas, and spleen of Jinhua pigs. The indicated tissue segments were collected from 6 Jinhua pigs at 270-day old following by RNA isolation, reverse transcription and RT-qPCR. Data was expressed as mean \pm SEM $(n=6)$. 
$\mathbf{A}$

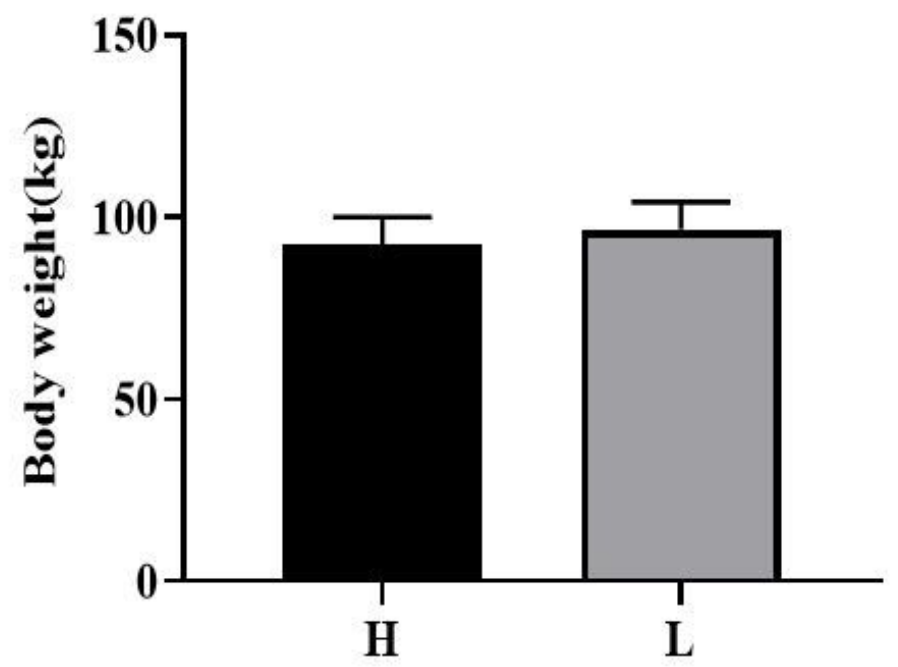

B

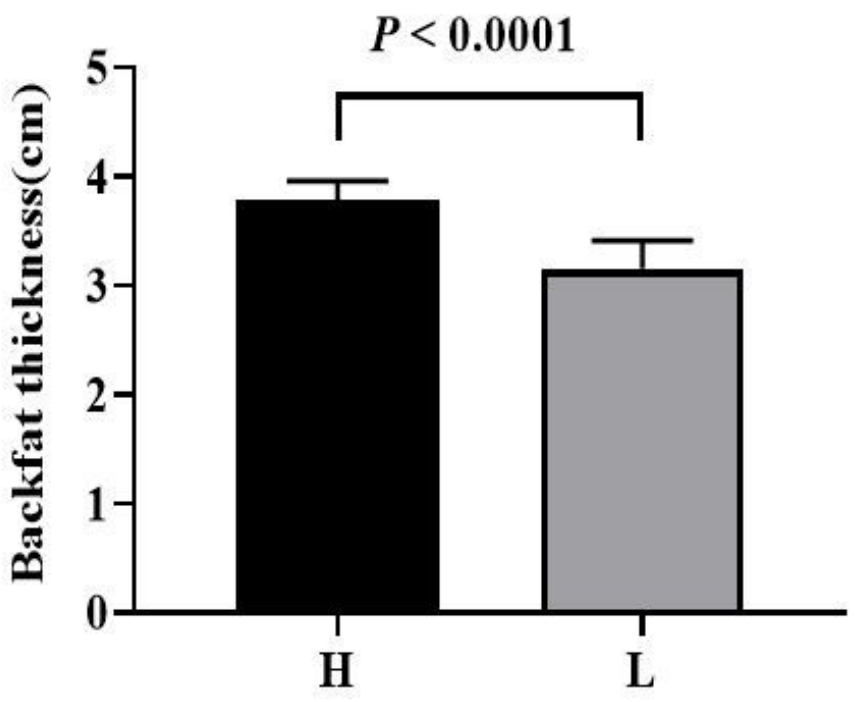

Figure 7

Body weight (A) and backfat thickness (B) of Jinhua pigs in the $\mathrm{H}$ and $\mathrm{L}$ groups. Jinhua pigs with similar body weight and significantly different backfat thickness were weighed individually and the backfat thickness of each pig was measured at 270-day old. $\mathrm{H}$ : the $\mathrm{H}$ group was consisted of pigs with relative high backfat thickness; $\mathrm{L}$ : the $\mathrm{L}$ group was consisted of pigs with relative low backfat thickness. Data was expressed as mean \pm SEM $(n=8)$ and analyzed by oneway ANOVA analysis followed by an unpaired two-tailed Student's t-test. 
$\mathbf{A}$

Liver

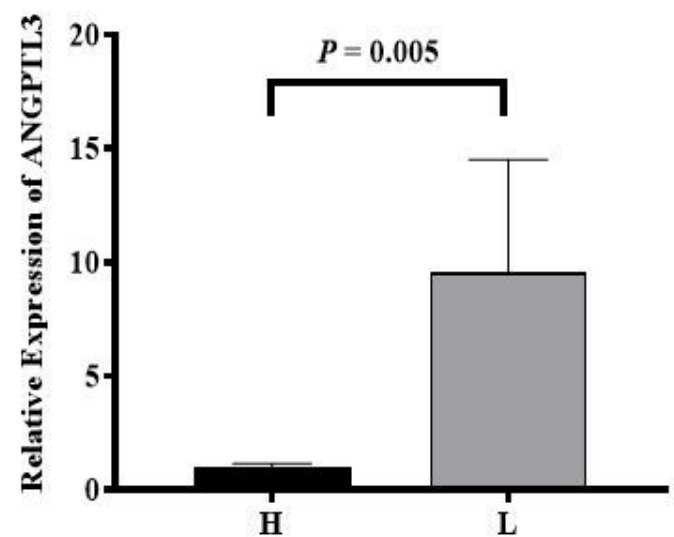

C

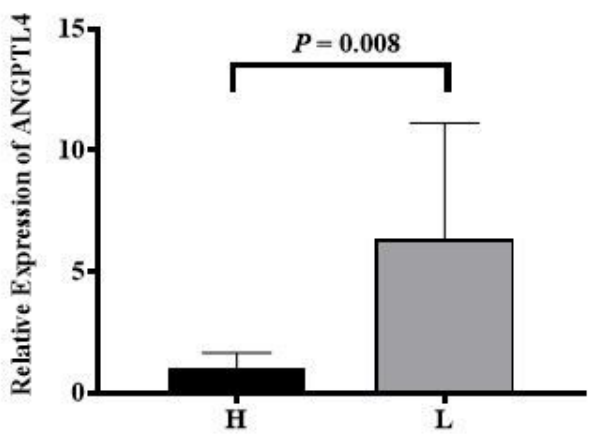

Liver

$\mathbf{H}$
B Liver

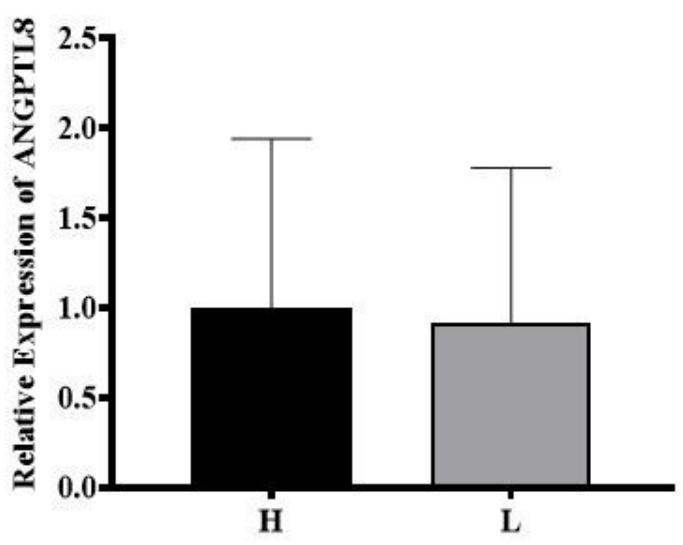

D

Subcutaneous Fat
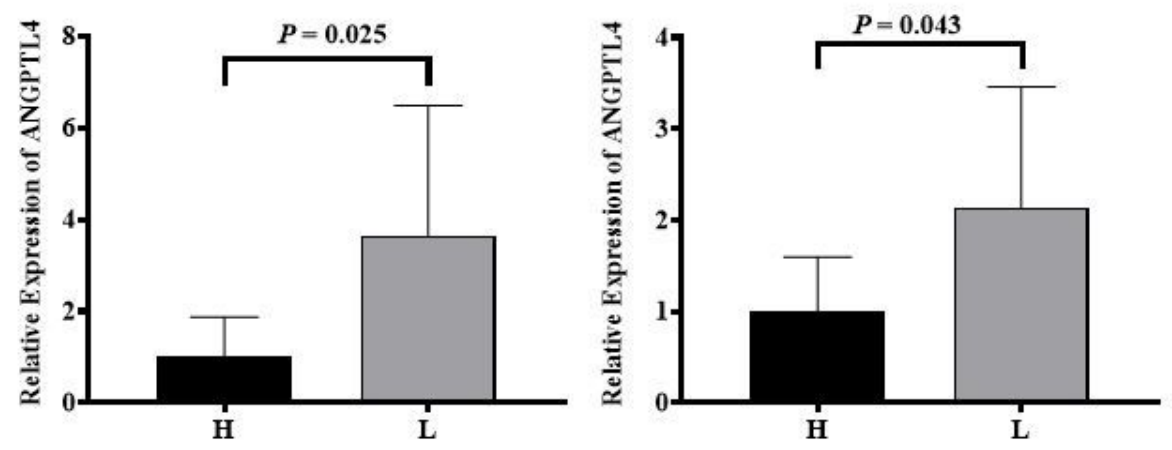

Figure 8

The expression of ANGPTL3, ANGPTL4, and ANGPTL8 in the liver (A, B, and C), subcutaneous fat (D), and ileum (E) of Jinhua pigs in the $H$ and $L$ groups. The liver, subcutaneous fat, and ileum segments were collected from 16 Jinhua pigs at 270-day old following by RNA isolation, reverse transcription and RT-qPCR. $\mathrm{H}$ : the $\mathrm{H}$ group was consisted of pigs with relative high backfat thickness; $L$ : the $L$ group was consisted of pigs with relative low backfat thickness. Data was expressed as mean \pm SEM $(n=8)$ and analyzed by one-way ANOVA analysis followed by an unpaired two-tailed Student's t-test. 